\title{
Survivin Deregulation in $\beta$-Tubulin Mutant Ovarian Cancer Cells Underlies Their Compromised Mitotic Response to Taxol
}

\author{
Jun Zhou, ${ }^{1,2}$ Aurora O’Brate, ${ }^{1}$ Amelia Zelnak, ${ }^{1}$ and Paraskevi Giannakakou' ${ }^{1}$ \\ ${ }^{1}$ Winship Cancer Institute and ${ }^{2}$ Department of Cell Biology, Emory University School of Medicine, Atlanta, Georgia
}

\section{ABSTRACT}

Taxol is one of the most successful drugs for the treatment of cancer because of its ability to target tubulin, block cell cycle progression at mitosis, and induce apoptosis. Despite the success of Taxol, the development of drug resistance hampers its clinical applicability. Herein we report that $\beta$-tubulin mutant, Taxol-resistant ovarian cancer cells exhibit defective mitotic response to Taxol, even at high concentrations that are sufficient to trigger apoptosis. This mitotic response-defective phenotype is independent of p53 status. We have found that survivin, the mitosis regulator and inhibitor of apoptosis protein, is deregulated in these Taxolresistant cancer cells; Taxol fails to induce survivin levels and survivin phosphorylation in these cells, in contrast to their parental drug-sensitive counterparts. Exogenous expression of wild-type survivin is able to restore the mitotic response of the resistant cells to Taxol treatment. On the other hand, exogenous expression of dominant-negative survivin abrogates the Taxol-induced mitotic response in drug-sensitive cancer cells. We have also found that overexpression of the mitotic kinase Cdk1, which phosphorylates survivin, is unable to restore the Taxol-induced mitotic response in the resistant cells. Our results show the importance of survivin for the mitotic response in the context of Taxol resistance and provide novel insights into the mechanisms of mitotic arrest and apoptosis induced by microtubule-targeting agents.

\section{INTRODUCTION}

Microtubules are essential components of the cytoskeleton and play a critical role in a variety of cellular processes including cell division, cell motility, intracellular trafficking, and cell shaping. Composed of $\alpha \beta$-tubulin heterodimers, microtubules are intrinsically dynamic polymers, and their dynamic property is crucial for the assembly of the mitotic spindle, the attachment of chromosomes to spindle microtubules, and the movement of chromosomes along the spindle (1-3). Suppression of microtubule dynamics by microtubule-targeting drugs such as the Vinca alkaloids and taxanes can engage the mitotic spindle checkpoint, arresting cell cycle progression at mitosis and eventually leading to apoptosis $(4,5)$.

Taxol is a complex diterpene derived from the Pacific yew tree Taxus brevifolia and binds to $\beta$-tubulin in a deep hydrophobic cleft near the luminal surface of microtubules $(6,7)$. At relatively high concentrations, Taxol promotes microtubule polymerization and increases microtubule-polymer mass (8). At lower concentrations (e.g., $1-10 \mathrm{nmol} / \mathrm{L}$ in HeLa cells), Taxol suppresses microtubule dynamics without significantly affecting polymer mass, retaining, however, its ability to induce mitotic arrest $(9,10)$.

Taxol is one of the most effective chemotherapeutic drugs to date, used clinically for the treatment of many solid tumors including ovarian, breast, prostate and non-small-cell lung cancer, and its clin-

Received 7/15/04; revised 8/23/04; accepted 9/30/04.

Grant support: This work was supported in part by a grant from the NIH (1R01 CA100202), a grant from Georgia Cancer Coalition, and an award from Aventis Pharmaceuticals to P. Giannakakou.

The costs of publication of this article were defrayed in part by the payment of page charges. This article must therefore be hereby marked advertisement in accordance with 18 U.S.C. Section 1734 solely to indicate this fact.

Requests for reprints: Paraskevi Giannakakou, Winship Cancer Institute, Emory University School of Medicine, Atlanta, GA 30322. Phone: 404-778-7729; Fax: 404-7785530; E-mail: pgianna@emory.edu.

(C)2004 American Association for Cancer Research. ical indications continue to expand (11). The applicability of Taxol has been seriously limited, however, by the development of drug resistance. The main mechanisms underlying Taxol resistance include defective intracellular drug accumulation mediated by overexpression of the drug efflux pump, P-glycoprotein (12), and microtubule-related mechanisms such as mutations in the $\beta$-tubulin gene $(13,14)$, differential expression of $\beta$-tubulin isotypes (15), and altered microtubule dynamics (16).

We have previously established a model of Taxol resistance consisting of a Taxol-sensitive human ovarian cancer cell line 1A9 and two Taxol-resistant derivative cell lines, namely PTX10 and PTX22 (13). These two derivative cell lines are 25-fold resistant to Taxol because of acquired $\beta$-tubulin mutations in the Taxol-binding site. Despite the 25-fold resistance, these Taxol-resistant cells undergo apoptosis when treated with higher, physiologically relevant, concentrations of Taxol. Herein we report that unlike the parental 1A9 cells, these Taxol-resistant cells have defective mitotic response to even high concentrations of Taxol that are sufficient to induce apoptosis. We also show that the defective mitotic response to Taxol in the Taxol-resistant cells is mediated by the deregulation of the mitosis regulator and inhibitor of apoptosis protein survivin (17). Our data show a previously unrecognized consequence of long-term exposure to Taxol and appreciate the importance of survivin in regulating cell division and cell survival in the presence of microtubule-targeting drugs.

\section{MATERIALS AND METHODS}

Materials. Taxol and vinblastine (Sigma-Aldrich, St. Louis, MO), Taxotere (Aventis Pharmaceuticals, Bridgewater, NJ), and epothilone B (Novartis, New York, NY) were from the indicated sources. All drugs were prepared as $100 \mathrm{mmol} / \mathrm{L}$ stocks in DMSO and stored in aliquots at $-80^{\circ} \mathrm{C}$. The following antibodies were used: rabbit anticleaved caspase 9 (p37), mouse anti-p21 and rabbit anti-Cdk1 (Cell Signaling, Beverly, MA); rabbit anticleaved poly(ADPribose) polymerase (p85, Promega, Madison, WI); mouse anti-p53, rabbit antisurvivin and goat antiphosphorylated survivin (Santa Cruz Biotechnology, Santa Cruz, CA); mouse anti- $\beta$-actin (Sigma-Aldrich); and rat anti- $\alpha$-tubulin (Chemicon, Temecula, CA). The following secondary antibodies were used: horseradish peroxidase-conjugated antirabbit and antimouse antibodies (Sigma-Aldrich), and rhodamine-conjugated antirat and fluorescein-conjugated antirabbit antibodies (Jackson ImmunoResearch, West Grove PA).

Cell Culture and Synchronization. The human ovarian carcinoma cell line 1A9 and its Taxol-resistant derivative cell lines were cultured in RPMI 1640 (Invitrogen, Carlsbad, CA) supplemented with $10 \%$ fetal bovine serum at $37^{\circ} \mathrm{C}$ in a humidified atmosphere with $5 \% \mathrm{CO}_{2} / 95 \%$ air. For experiments involving synchronization, cells were grown in culture medium containing $1 \%$ fetal bovine serum for 30 hours (serum starvation) and then replaced with medium containing $10 \%$ fetal bovine serum (serum add back). The percentage of cells in $\mathrm{S}$ phase at different time points after serum add back was determined with a 5-bromo-2'-doxyuridine (BrdUrd) labeling and detection kit (Roche Applied Science, Indianapolis, IN).

Flow Cytometry. Analysis of the cell cycle by fluorescence-activated cell sorting assay was done as described previously (18). In brief, cells were centrifuged, washed twice with ice-cold PBS, and fixed in $70 \%$ ethanol at $-20^{\circ} \mathrm{C}$. Cells were resuspended in $30 \mu \mathrm{L}$ of phosphate/citrate buffer $[0.2$ $\mathrm{mol} / \mathrm{L} \mathrm{Na} \mathrm{HPO}_{4} / 0.1 \mathrm{~mol} / \mathrm{L}$ citric acid $\left.(\mathrm{pH} 7.5)\right]$ at room temperature for 30 minutes, and then incubated with propidium iodide $(20 \mu \mathrm{g} / \mathrm{mL}) / \mathrm{RNase}$ A (20 
$\mu \mathrm{g} / \mathrm{mL}$ ) in PBS for 30 minutes. Samples were analyzed on a Coulter Elite flow cytometer (Beckman Coulter, Fullerton, CA).

Transient Transfection and Adenovirus Infection. Transient transfections were done with the Fugene 6 reagent (Roche Applied Science), as described previously (19). The replication-deficient $\beta$-galactosidase and p53 adenoviruses were kind gifts from Dr. Bert Vogelstein (Johns Hopkins University) and amplified in low passage HEK293 cells. Adenovirus titers were determined by with an adenovirus titer kit (BD Biosciences, San Jose, CA). The multiplicity of infection was defined as the ratio of infectious units divided by the number of cells.

Time-Lapse Video Microscopy. We examined cells grown in 35-mm dishes with a Zeiss Axiovert microscope equipped with phase-contrast, using a $20 \times$ objective (Zeiss, Thornwood, NY). A stage heater was used to maintain the temperature at $37 \pm 0.5^{\circ} \mathrm{C}$. Images were taken with a Hamamatsu Orca II camera (Hamamatsu, Middlesex, NJ) every 4 minutes controlled by Metamorph image analysis software (Universal Imaging, Downingtown, PA).

Fluorescence Microscopy. Cellular survivin and microtubules were examined by immunofluorescence microscopy as described previously $(20,21)$. In brief, cells grown on 12-mm glass coverslips were fixed with PHEMO buffer [68 mmol/L PIPES, $25 \mathrm{mmol} / \mathrm{L}$ HEPES, $15 \mathrm{mmol} / \mathrm{L}$ EGTA, $3 \mathrm{mmol} / \mathrm{L} \mathrm{MgCl}_{2}$, $10 \%$ DMSO (pH 6.8)] containing $3.7 \%$ formaldehyde, $0.05 \%$ glutaraldehyde, and $0.5 \%$ Triton X-100 for 10 minutes at room temperature. Nonspecific sites were blocked by incubating with $10 \%$ goat serum in PBS for 10 minutes and processed for immunofluorescence staining with rat anti- $\alpha$-tubulin and rabbit antisurvivin antibodies. The secondary antibodies were rhodamine-conjugated antirat and fluorescein-conjugated antirabbit antibodies. We then mounted coverslips onto glass slides and examined them with a Zeiss axioplasm laser scanning confocal microscope using a Zeiss $100 \times 1.3$ oil-immersion objective. For experiments involving quantification of mitotic and apoptotic cells, cells were stained with propidium iodide and the morphological changes in the nuclear chromatin of cells were then examined with a Zeiss Axiovert microscope.

Determination of Cdk1 Activity. Cdk1 activity was measured by the histone $\mathrm{H} 1$ kinase assay kit (Upstate Biotechnology, Lake Placid, NY). Briefly, cell lysates were prepared in $20 \mathrm{mmol} / \mathrm{L}$ Tris ( $\mathrm{pH} \mathrm{7.4),} 200 \mathrm{mmol} / \mathrm{L} \mathrm{NaCl}, 1 \%$ NP40 with protease inhibitor mixture (Roche Applied Science). The protein concentrations were determined by bicinchoninic acid protein assay (Pierce Biotechnology, Rockford, IL). To immunoprecipitate Cdk1, cell lysate containing $100 \mu \mathrm{g}$ of total protein was incubated with Cdk1 antibody in excess and protein A/G-agarose beads at $4^{\circ} \mathrm{C}$ overnight. The $\mathrm{Cdk} 1$ immunoprecipitates were washed three times with the lysis buffer and then used for the histone $\mathrm{H} 1$ kinase assay.

Western Blotting. Proteins extracted from cells were resolved by SDSPAGE and electrophoretically transferred onto polyvinylidene difluoride membranes (Millipore, Bedford, MA). The membranes were blocked for 2 hours in Tris-buffered saline containing $0.2 \%$ Tween 20 and 5\% fat-free dry milk, and then incubated first with primary antibodies and then horseradish peroxidaseconjugated secondary antibodies for 2 and 1 hour, respectively. Specific proteins were visualized with enhanced chemiluminescence detection reagent following the manufacturer's instructions (Pierce Biotechnology). The intensity of protein bands was determined by densitometric analysis with a Lynx video densitometer (Biological Vision, Inc., San Mateo, CA).

\section{RESULTS}

High Concentrations of Taxol Induce Apoptosis in the Absence of Mitotic Arrest in Taxol-Resistant, $\beta$-Tubulin Mutant Cancer Cells. The Taxol-resistant, $\beta$-tubulin mutant human ovarian cancer cell line PTX10 exhibits 25-fold resistance to Taxol as compared with the parental Taxol-sensitive 1A9 cells (13). Cell cycle analysis (fluorescence-activated cell sorting) revealed that whereas 1A9 cells underwent mitotic arrest when treated for 16 hours with as little as 10 $\mathrm{nmol} / \mathrm{L}$ of Taxol, PTX10 cells failed to arrest in mitosis even when treated with 100-fold higher concentrations of Taxol $(1 \mu \mathrm{mol} / \mathrm{L}$; Fig. 1A). Nevertheless, both $1 \mathrm{~A} 9$ and PTX10 cells exhibited apoptotic cell death in response to Taxol, as indicated by caspase 9 and poly(ADPribose) polymerase cleavage (Fig. $1 B$ ).

To better understand the mechanism by which Taxol induced

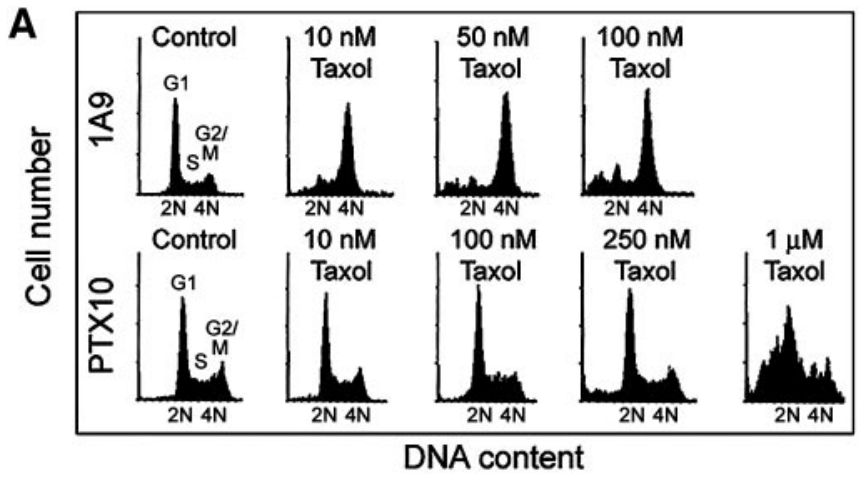

B

$1 \mathrm{~A} 9$
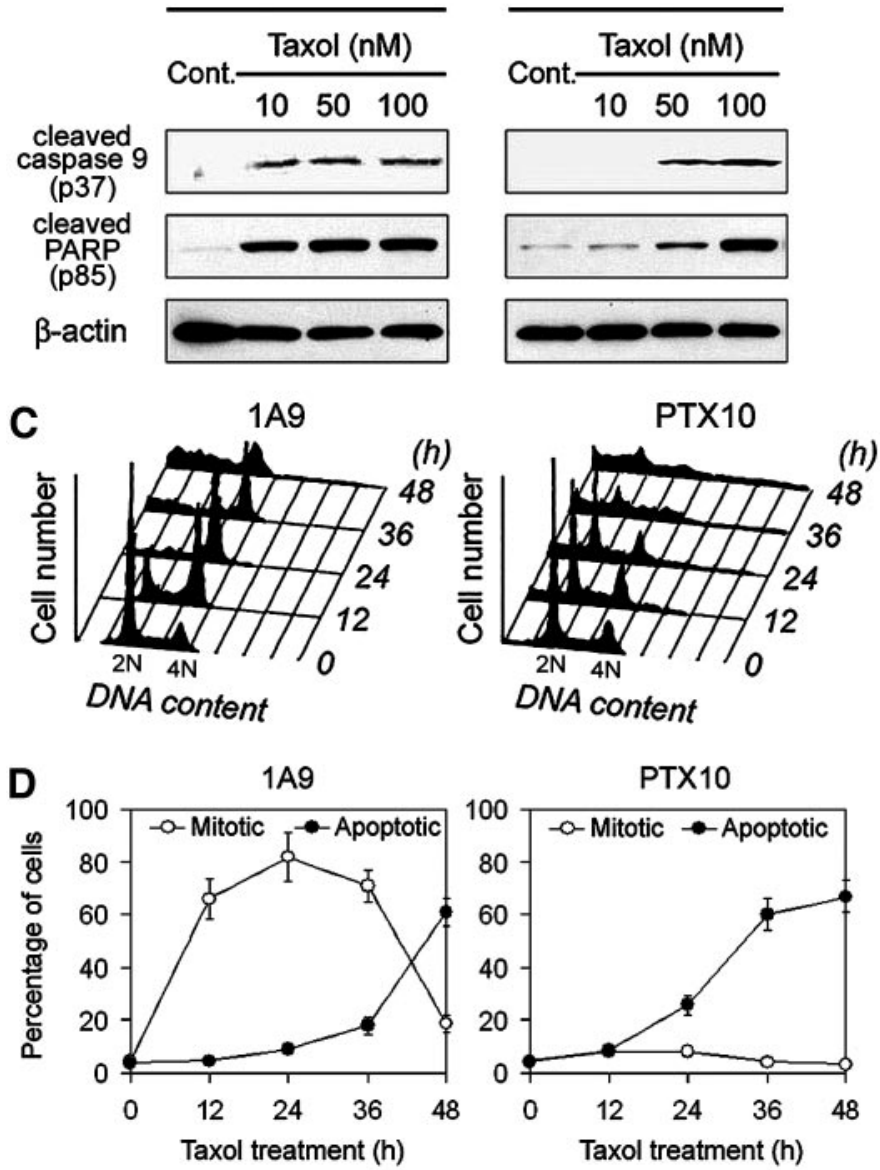

Fig. 1. Differential mitotic response to Taxol in Taxol-sensitive and Taxol-resistant ovarian cancer cells. A, fluorescence-activated cell sorting analysis of DNA content in the Taxol-sensitive cell line 1A9 and in the Taxol-resistant derivative cell line PTX10. Cells were treated for 16 hours with the indicated concentrations of Taxol or with the solvent DMSO as control. Cells with $2 \mathrm{~N}$ DNA content are in $\mathrm{G}_{1}$ phase, 2 to $4 \mathrm{~N}$ in $\mathrm{S}$ phase, and $4 \mathrm{~N}$ in $\mathrm{G}_{2}-\mathrm{M}$ phase. $B$, Western blot analysis of cleaved caspase 9 (p37) and cleaved poly(ADP-ribose) polymerase (p85) in 1A9 and PTX10 cells treated with Taxol for 16 hours. $C$, time course fluorescence-activated cell sorting analysis of DNA content in 1A9 and PTX10 cells treated with 10 and $250 \mathrm{nmol} / \mathrm{L}$ Taxol, respectively. $D$, percentages of mitotic and apoptotic cells measured by DNA staining. 1A9 and PTX10 cells were treated with 10 and $250 \mathrm{nmol} / \mathrm{L}$ Taxol, respectively, for the indicated time. The values and error bars shown in this figure and in the following figures represent the averages and SDs, respectively, of three independent experiments.

apoptosis in PTX10 cells without prior mitotic arrest, we did a time course cell cycle analysis of 1A9 and PTX10 cells treated with 10 and $250 \mathrm{nmol} / \mathrm{L}$ Taxol, respectively. These concentrations were selected based on the fact that PTX10 cells are 25-fold resistant to Taxol as compared with $1 \mathrm{~A} 9$ cells. As shown in Fig. 1C, 1A9 cells showed mitotic arrest as early as 12 hours after Taxol treatment; however, no 
A
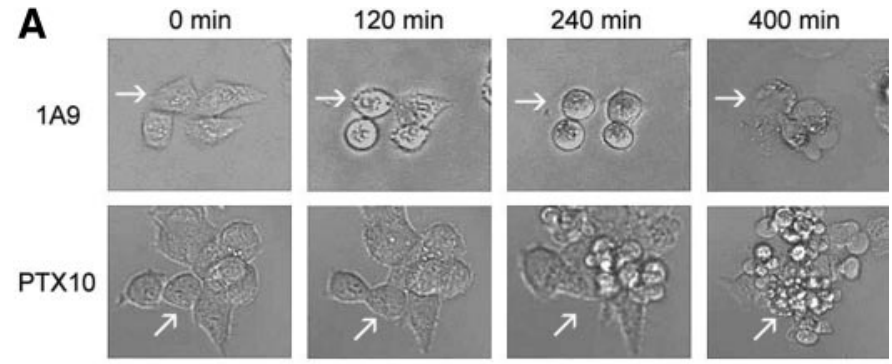

\section{B}

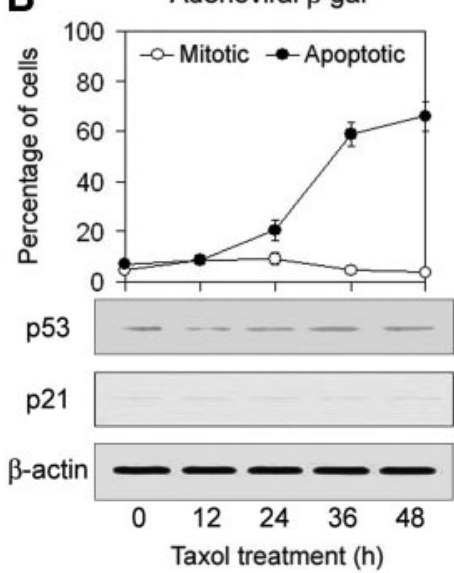

Adenoviral p53

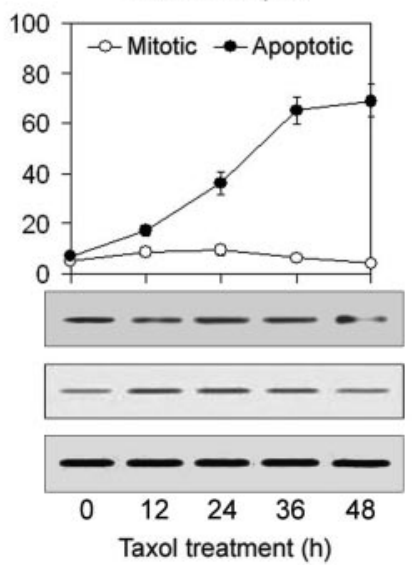

Fig. 2. The lack of mitotic arrest before apoptosis in PTX10 cells is independent of p53 status. A, time-lapse video microscopy of 1 A9 and PTX10 cells treated with 10 and 250 $\mathrm{nmol} / \mathrm{L}$ Taxol, respectively. Arrows point to representative cells. B, PTX10 cells were infected with 10 multiplicity of infection adenoviral $\beta$-galactosidase $(\beta$-gal) or p53 and then treated with $250 \mathrm{nmol} / \mathrm{L}$ Taxol for $0,12,24,36$, or 48 hours. Note that although adenoviral p53 can restore p 1 induction in PTX10 cells, it does not enhance mitotic arrest in response to Taxol.

obvious mitotic arrest was observed in PTX10 cells even after 48 hours of Taxol treatment. Figure $1 D$ shows the percentages of mitotic and apoptotic cells in response to the treatment of Taxol $(10 \mathrm{nmol} / \mathrm{L}$ for $1 \mathrm{~A} 9$ and $250 \mathrm{nmol} / \mathrm{L}$ for PTX10). For example, almost complete mitotic arrest (82\%) was observed in 1A9 after 24 hours of treatment with Taxol; in contrast, a very modest mitotic arrest (8\%) was observed in PTX10 cells after Taxol treatment. Nevertheless, after 48 hours of treatment, 1A9 and PTX10 cells exhibited a similar extent of apoptosis, 61 and $67 \%$, respectively.

The lack of mitotic arrest before apoptosis in PTX10 cells was further confirmed by time-lapse video microscopy (Fig. 2A). In this experiment, living 1A9 and PTX10 cells were visualized in the presence of 10 and $250 \mathrm{nmol} / \mathrm{L}$ Taxol, respectively. An obvious mitotic arrest was observed in 1A9 cells (cells rounding up) as early as 120 minutes of drug treatment. In contrast, no mitotic arrest was observed in PTX10 cells even after 400 minutes of drug treatment. PTX10 cells exhibited apoptotic figures after 240 minutes of Taxol exposure whereas $1 \mathrm{~A} 9$ cells underwent apoptosis clearly after mitotic arrest (Fig. 2A).

The Lack of Mitotic Arrest in PTX10 Cells Is Independent of p53 Status. The Taxol-resistant PTX10 cells have acquired a p53 point mutation, in addition to the acquired $\beta$-tubulin mutation (22). Because p53 function has been implicated in the control of mitotic progression (23), we investigated whether the mutant p53 status contributed to the lack of mitotic arrest in PTX10 cells in response to Taxol. Thus, PTX10 cells were infected with p53 adenoviruses or control $\beta$-galactosidase viruses and then treated with $250 \mathrm{nmol} / \mathrm{L}$ of Taxol (Fig. 2B). Exogenous expression of p53 resulted in a robust up-regulation of $\mathrm{p} 21$; however, it did not restore the ability of Taxol to induce mitotic arrest in these cells. When a similar experiment was done in the parental 1A9 cells, exogenous expression of p53 did not further induce the mitotic response to Taxol treatment (data not shown). This result indicated that the lack of mitotic arrest in PTX10 cells was independent of p53 status.

PTX10 Cells Undergo Very Transient Mitotic Arrest in Response to Taxol. To further investigate the molecular mechanism underlying the lack of Taxol-induced mitotic arrest in PTX10 cells, we synchronized cells by serum starvation and examined their cell cycle progression by BrdUrd incorporation assay. As shown in Fig. $3 A$, both 1A9 and PTX10 cells exhibited a peak of BrdUrd-positive cells 9.5 hours after serum add back indicating $S$ phase, when cells are active in DNA synthesis. We asked at which stage of the cell cycle Taxol-induced apoptosis occurred, by treating cells with Taxol (10 $\mathrm{nmol} / \mathrm{L}$ for $1 \mathrm{~A} 9$ and $250 \mathrm{nmol} / \mathrm{L}$ for PTX10) at 4, 9.5, or 15 hours after serum add back. These time points correlated with $G_{1}, S$, or $G_{2}$ phase of the cell cycle, respectively. As shown in Fig. 3B, 1A9 and PTX10 cells both showed the highest mitotic indices at 19 hours after serum add back, indicating mitosis. In addition, 1A9 and PTX10 cells treated with Taxol at any phase of the cell cycle $\left(G_{1}, S\right.$, or $\left.G_{2}\right)$ entered mitosis (Fig. 3C and data not shown).

However, whereas 1A9 cells exhibited a prolonged ( $>18$ hours) mitotic arrest before apoptosis, PTX10 cells showed only a transient $(<3$ hours) mitotic arrest (Fig. $3 C$ and $D)$. The differential mitotic responses of 1A9 and PTX10 cells were also observed when higher concentrations of Taxol were used. For example, 1A9 cells remained able to arrest at mitosis for $>15$ hours in response to even $1 \mu \mathrm{mol} / \mathrm{L}$ Taxol. However, PTX10 cells never exhibited mitotic arrest for $>3$ hours. These data collectively indicated that Taxol-induced apoptosis occurred after entry to mitosis in both 1A9 and PTX10 cells, regardless of the time they spent in mitosis. To examine whether the compromised mitotic response of PTX10 cells was Taxol specific or could apply to other drugs that induce mitotic arrest, we examined PTX10 mitotic response to additional microtubule inhibitors (Fig. $3 D$ ). A similar pattern of transient mitotic arrest in PTX10 cells was observed after treatment with the microtubule-stabilizing drugs Taxotere and epothilone $\mathrm{B}$ and with the microtubule-destabilizing drug vinblastine (Fig. 3D). In addition, another Taxol-resistant ovarian cancer cell line, PTX22, showed a pattern of transient mitotic arrest similar to that observed in the PTX10 cell line (data not shown). These results therefore suggested that the mitotic response was severely impaired in these Taxol-resistant ovarian cancer cells as compared with the parental Taxol-sensitive cells.

Deregulation of Survivin in Response to Taxol Treatment in the Taxol-Resistant Cancer Cells. To study the mechanism by which the Taxol-resistant cells exhibited defective mitotic response to Taxol, we investigated several major proteins involved in the control of mitotic progression, including the mitotic spindle checkpoint proteins Mad2, Bub1, and BubR1 (24), and the mitosis regulator and inhibitor of apoptosis protein survivin (17). There was no significant difference in Mad2, Bub1, and BubR1 protein levels between 1A9 and PTX10 cells (data not shown). Taxol, which is known to increase survivin levels (25), failed to induce survivin in PTX10 cells compared with the robust induction observed in the parental 1A9 cells (Fig. 4). For example, survivin expression was increased up to 7.4-fold in synchronized, Taxol-treated 1A9 cells. In contrast, there was no obvious increase in survivin level in PTX10 cells.

To examine whether in PTX10 cells the deregulation of survivin was attributable to incorrect intracellular localization, we stained cellular survivin and examined with confocal fluorescence microscopy (Fig. 5). No difference was observed in survivin localization patterns between mitotic 1A9 and PTX10 cells. However, this experiment further showed a lack of survivin induction in PTX10 cells in response to Taxol treatment (Fig. 5). 
Fig. 3. PTX10 cells undergo very transient mitotic arrest in response to Taxol. A, 1A9 and PTX10 cells were synchronized by serum starvation as described in Materials and Methods, and the percentage of cells in $\mathrm{S}$ phase after serum add back was determined by BrdUrd incorporation at the indicated time points. $B$, cells were synchronized by serum starvation, and the mitotic indices after serum add back were subsequently measured by DNA staining at the indicated time points. $C$, cells were synchronized by serum starvation, and 4 hours after serum add back, they were treated with 10 and $250 \mathrm{nmol} / \mathrm{L}$ Taxol, respectively. The mitotic indices were then measured at the indicated time points. $D$, $1 \mathrm{~A} 9$ and PTX10 cells were processed as in $C$, and the duration of mitotic arrest in response to various microtubule-targeting drugs was measured by DNA staining.
A

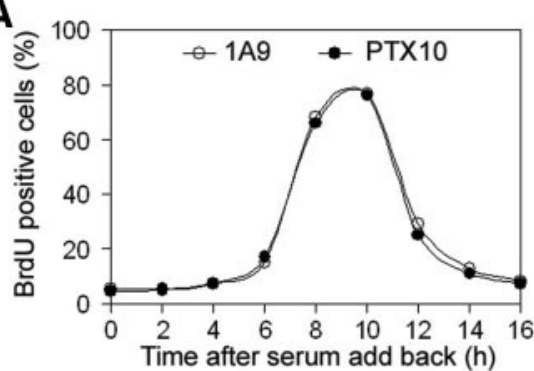

C

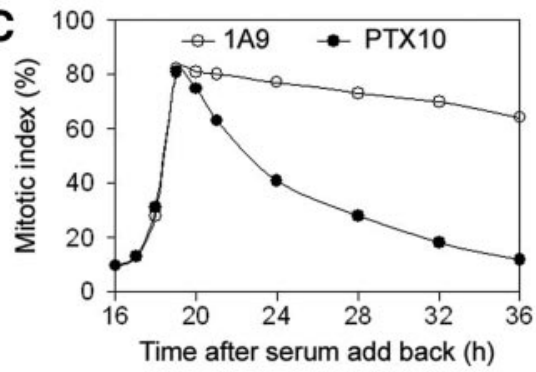

B
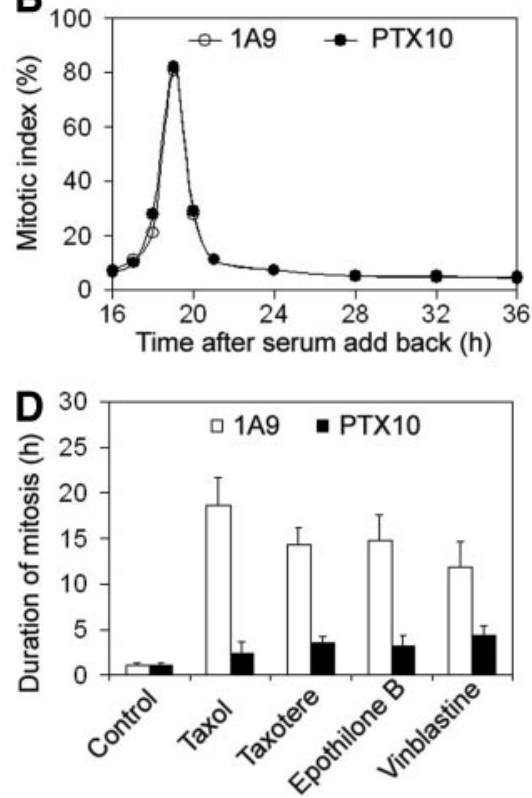

Survivin Plays an Important Role in the Mitotic Response to Taxol. To test whether the deregulation of survivin in the Taxolresistant cells contributed to their defective mitotic response to Taxol, we introduced exogenous wild-type survivin in PTX10 cells and examined the percentages of mitotic and apoptotic cells after Taxol treatment (Fig. 6A). We found that exogenous expression of wild-type survivin restored the mitotic response of PTX10 cells to Taxol treatment (the peak mitotic arrest was $62 \%$ at 24 hours of Taxol treatment; Fig. 6A). In contrast, expression of dominant-negative survivin had no effect on their mitotic response (Fig. 6A). In addition, exogenous expression of wild-type survivin had no obvious effect on the Taxolinduced mitotic response in 1A9 cells (Fig. 6B). However, when 1A9 cells were transfected with dominant-negative survivin, their mitotic response to Taxol was significantly suppressed to a level comparable with that observed in PTX10 cells (Fig. 6B). We also observed that exogenous expression of survivin could enhance the mitotic arrest of PTX22 cells to Taxol treatment (data not shown). These results thus showed the importance of survivin in the control of mitotic response to Taxol treatment. We noticed that transfection of dominant-negative survivin did not significantly enhance the apoptotic response to Taxol in 1A9 and PTX10 cells (Fig. 6), in contrast to the observation that survivin inhibition by small interfering RNA significantly increased Taxol-induced death in MCF-7 cells (26). The difference in the two studies may be attributable to the use of different cell lines or methods for survivin inhibition. Alternatively, it may result from the different assays used for the examination of apoptosis.

The kinase activity of Cdk1 has been implicated recently in the regulation of survivin expression and cell viability (25). Thus, we investigated whether Cdk1 also played a regulatory role upstream of survivin in the mitotic response of 1A9 and PTX10 cells to Taxol treatment. Our results revealed a sustained activation of Cdk1 in Taxol-treated 1A9 cells but only transient activation in PTX10 cells, consistent with the sustained mitotic arrest in 1A9 cells and transient mitotic arrest in PTX10 cells (Fig. 7A and $B$ ). Phosphorylation of survivin on $\mathrm{Thr}^{34}$ by Cdk1 was proposed to be critical for survivin stability (25). To test whether the transient Cdk1 activation also affected survivin phosphorylation and function in PTX10 cells, we assessed phospho- Thr $^{34}$ survivin levels after Taxol treatment (Fig. 7A and $C$ ). No induction in phospho- $\mathrm{Thr}^{34}$ survivin was observed in
Taxol-treated PTX10 cells. In contrast, Taxol treatment of 1A9 cells resulted in increased phospho-Thr ${ }^{34}$ survivin levels (Fig. 7A and $C$ ). To test the significance of Cdk1 in the defective mitotic response of PTX10 cells, we introduced exogenous Cdk1 in PTX10 cells and examined the percentages of mitotic and apoptotic cells after Taxol treatment (Fig. 7D). Interestingly, expression of Cdk1 had no significant effect on the mitotic response of PTX10 cells to Taxol (Fig. 7D). Transfection of Cdk1 did not affect the mitotic response of 1A9 cells, either (Fig. 7E). These results suggested that Cdk1 might play a minor role if any in the defective mitotic response of PTX10 cells to Taxol, although its activity was clearly required to prevent exit from Taxolinduced mitotic arrest.
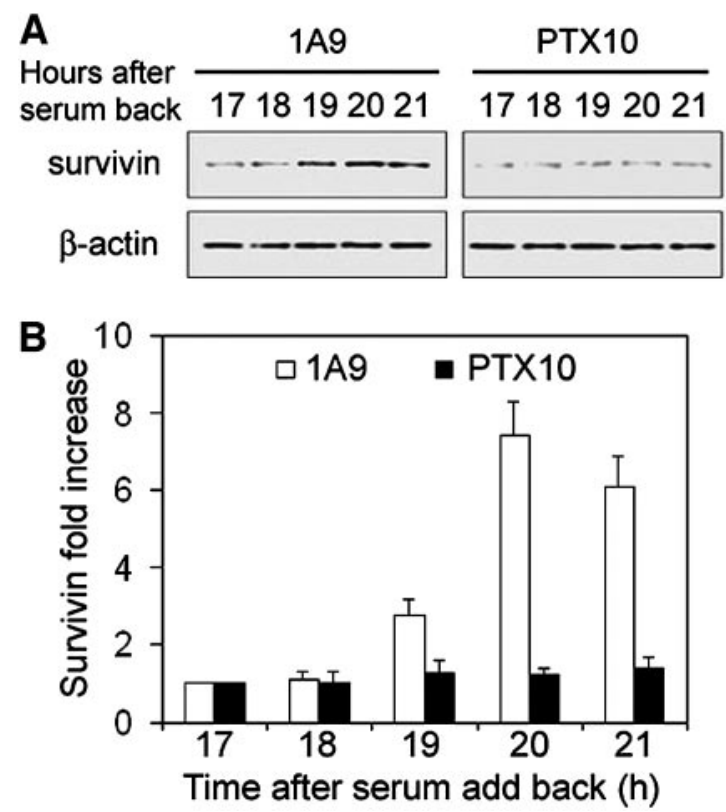

Fig. 4. Deregulation of survivin in response to Taxol in PTX10 cells. A, survivin levels analyzed by Western blotting. 1A9 and PTX10 cells were synchronized by serum starvation, and 4 hours after serum add back, they were treated with 10 and $250 \mathrm{nmol} / \mathrm{L}$ Taxol respectively, for the indicated time. Actin was used as a loading control. $B$, fold increase of survivin in response to Taxol. Experiments were done as in $A$, and survivin levels were determined by densitometric analysis of the protein bands on Western blots. 
$1 \mathrm{~A} 9$
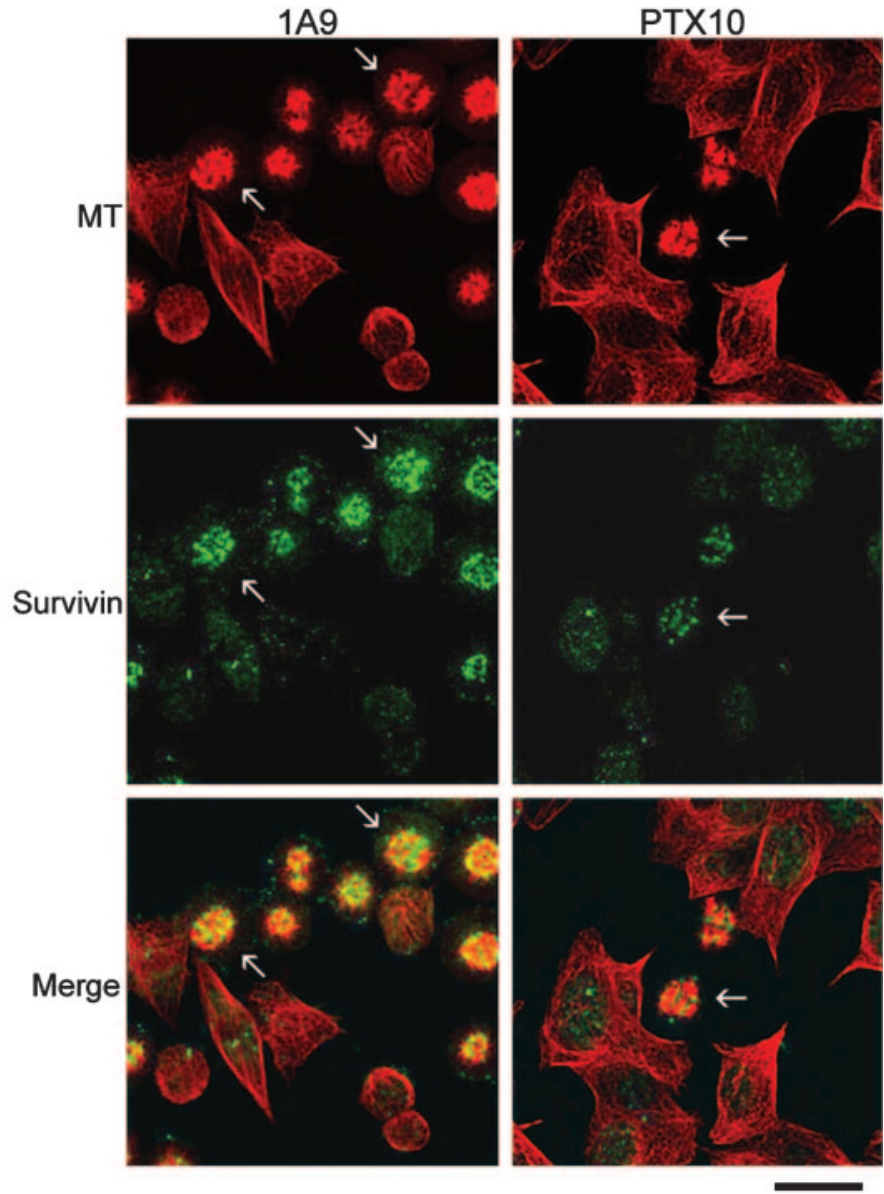

Fig. 5. Immunofluorescence images of microtubules (MT, red) and survivin (green) in $1 \mathrm{~A} 9$ and PTX10 cells treated for 12 hours with 10 and $250 \mathrm{nmol} / \mathrm{L}$ Taxol, respectively. Arrows point at representative mitotic cells. Bar, $10 \mu \mathrm{m}$.

\section{DISCUSSION}

Drug resistance is a major problem that hampers the long-term effectiveness of Taxol in cancer chemotherapy. In fact, the majority of cancer death is attributable to acquired drug resistance in the clinic. Thus, understanding of the molecular mechanisms contributing to drug resistance may provide us with the opportunity to develop more effective anticancer therapies. One of the mechanisms underlying Taxol resistance involves acquired $\beta$-tubulin mutations that interfere with drug-target binding. We have previously established a model of Taxol resistance by selecting the 1A9 human ovarian cancer cells with Taxol. As a result, two drug-resistant clones, PTX10 and PTX22, were obtained exhibiting 25 -fold resistance attributable to acquired $\beta$-tubulin mutations in the Taxol-binding site (13). In this study, we have shown that these Taxol-resistant cells undergo apoptosis when treated with higher, physiologically relevant Taxol concentrations, albeit without prior arrest in mitosis. In addition, we have found that the mitotic response of PTX10 cells to other microtubule-targeting drugs is also severely compromised, even in the absence of any cross-resistance (e.g., vinblastine), compared with the parental 1A9 cells. These results are very intriguing because the relationship between Taxol-induced mitotic arrest and apoptosis remains largely unknown (27). Mitotic arrest may provide a "sensitive" state that prepares cells for subsequent apoptosis. Alternatively, mitotic arrest and apoptosis may represent two independent events that occur sequentially, resulting from the disruption of the normal physiologic balance of microtubule dynamics. Our results show that even in the absence of a robust mitotic arrest, PTX10 cells are perfectly capable of undergoing apoptosis when treated with Taxol and other microtubule inhibitors, supporting the latter model of a temporal association between mitotic arrest and apoptosis.

To understand the mechanism underlying the compromised mitotic response in PTX10 cells, we have examined the potential involvement of proteins important for mitotic progression. Among the proteins examined, survivin seems to be the one critically involved in this pathway. Survivin has attracted considerable attention over the past years because of its dual role in mitotic progression and apoptosis. Furthermore, this small protein (16 kilodaltons) is highly overexpressed in many types of cancer but nearly undetectable in normal differentiated adult tissues and thus seems to be a bona fide target for cancer-specific therapy (17). At the cellular level, survivin is specifically expressed during the mitotic phase of the cell cycle (28). It has been reported that the up-regulated expression of survivin in mitosis
Fig. 6. Survivin plays an important role in the mitotic response to Taxol. PTX10 $(A)$ and 1A9 $(B)$ cells were transfected with plasmids expressing wild-type survivin (survivinwt), dominant-negative survivin (survivin-dn), or an empty vector, and 24 hours later they were treated with 250 and 10 $\mathrm{nmol} / \mathrm{L}$ Taxol, respectively. Percentages of mitotic and apoptotic cells were measured by DNA staining at the indicated time points after Taxol treatment.

\section{A PTX10 cells}
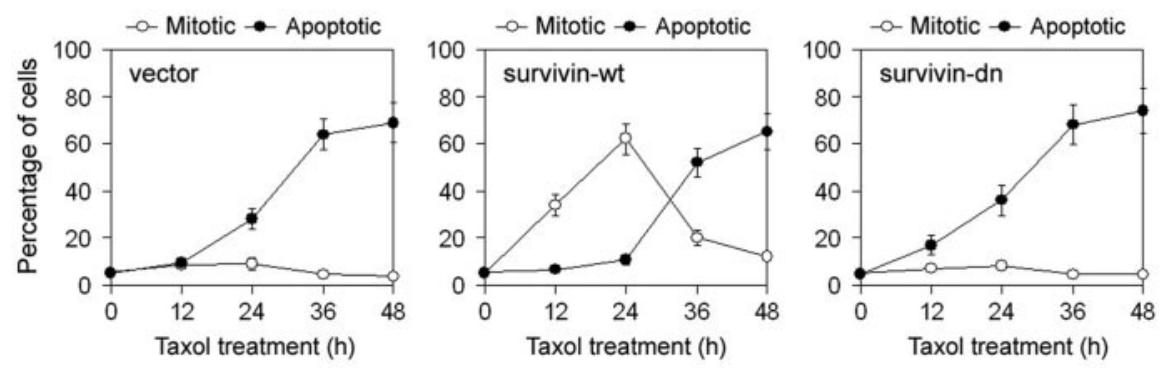

\section{B 1 A9 cells}
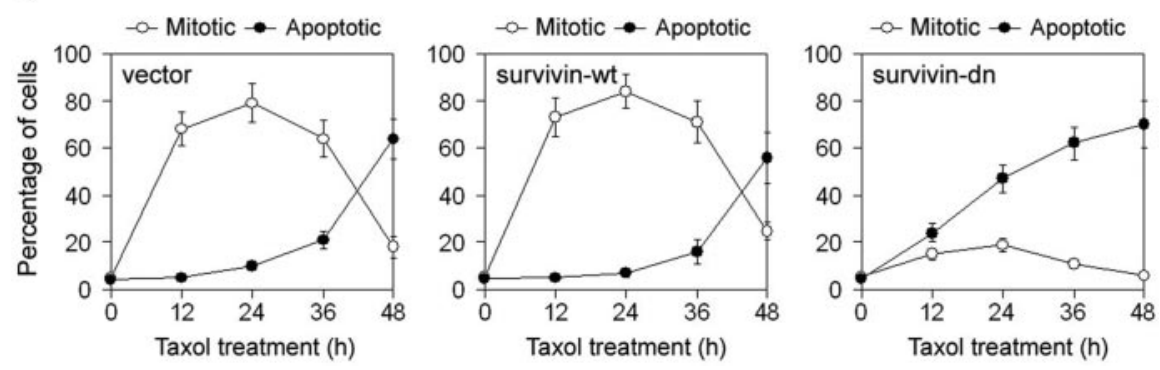


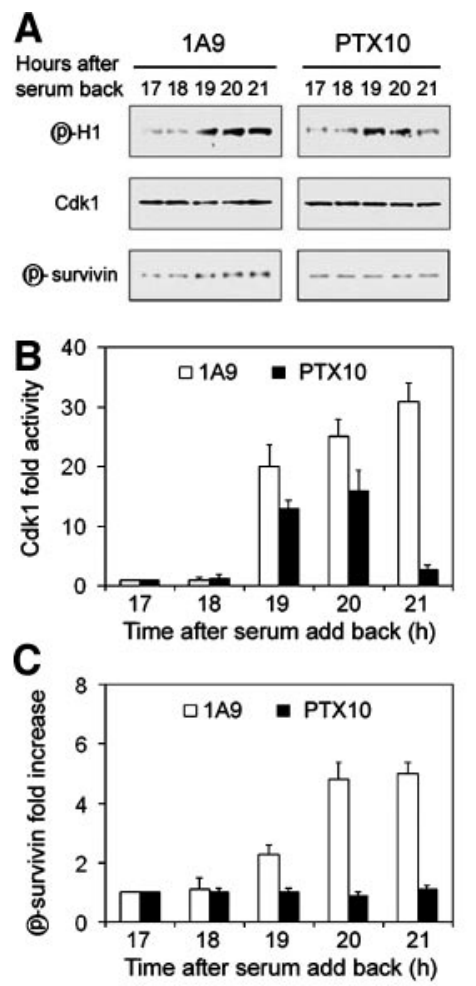

\section{PTX10 cells}
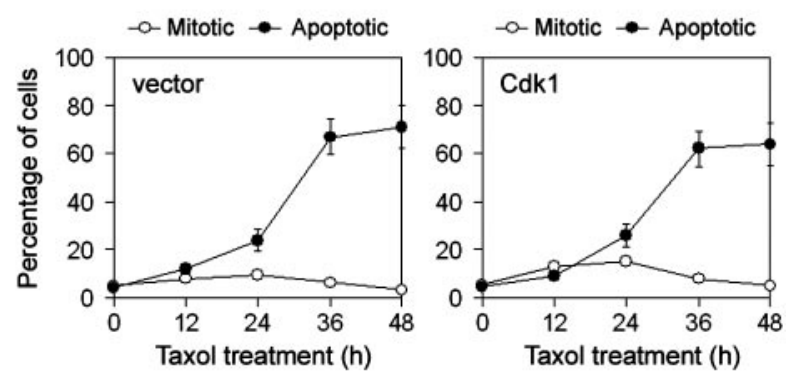

\section{E 1 A9 cells}
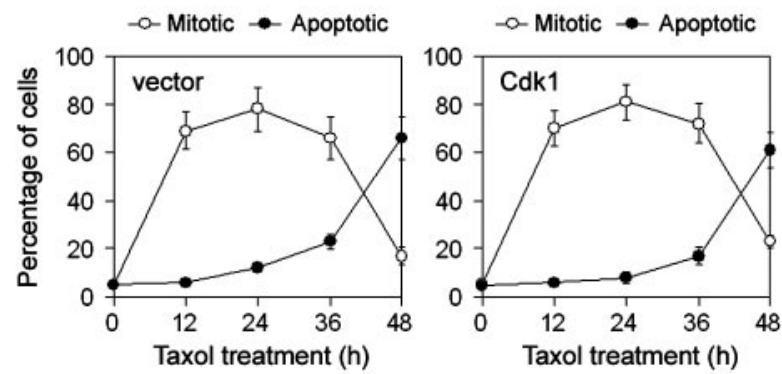

can be further induced by microtubule-targeting drugs in a dosedependent manner (25). We have also found that Taxol can increase survivin expression in 1A9 cells, but not in the Taxol-resistant PTX10 cells even at high concentrations that not only compensate for the 25 -fold resistance but also lead to cell death. Furthermore, exogenous expression of wild-type survivin is able to partially restore the mitotic response of PTX10 cells to Taxol, although this mitotic response is not as robust and sustained as in 1A9 cells. Interestingly, introduction of exogenous dominant-negative survivin in the parental 1A9 cells mimics the phenotype of PTX10 cells in terms of defective Taxolinduced mitotic response. Taken together, these results suggest that functional survivin is required for the effective mitotic response to Taxol. To investigate whether survivin gene mutation could account for the lack of survivin function in response to Taxol in PTX10 cells, we have sequenced survivin in both parental and resistant cells. In both cell lines, survivin gene sequence was identical with wild-type (data not shown), suggesting that survivin deregulation in PTX10 cells is not mediated by sequence alterations.

Our results suggest the involvement of survivin deregulation in mediating the compromised mitotic response to Taxol; however, at present we cannot rule out the involvement of deregulation of spindle checkpoint components in this process. The activities of many spindle checkpoint proteins are known to be regulated by phosphorylation, and a compromised spindle checkpoint often results in mitotic slippage. Thus, it is possible that deregulated spindle checkpoint components may also mediate the compromised mitotic response to Taxol in PTX10 cells, although there is no significant difference in the expression level of Mad2, Bub1, and BubR1 between 1A9 and PTX10 cells.

Sequential activation and inactivation of Cdk1 is required for cells to enter and exit mitosis, respectively. Cdk1-mediated phosphorylation of survivin on $\mathrm{Thr}^{34}$ has been shown recently to be critical for survivin function and stability $(25,29)$. We have shown that in the Taxol-resistant PTX10 cells, Cdk1 activity is only transiently activated by Taxol, coincident with the transient mitotic arrest. However, survivin phosphorylation on $\mathrm{Thr}^{34}$ was undetectable in these cells. In addition, exogenous expression of $\mathrm{Cdk} 1$ is unable to restore the mitotic response of PTX10 cells to Taxol, in sharp contrast to the effects of exogenous expression of survivin in these cells. These data together indicate that the compromised mitotic response in PTX10 cells might mainly result from the deregulation of survivin, which cannot be restored by Cdk1 level or activity. It is possible that other mitotic kinases (e.g., Aurora and Polo kinase families) also contribute to the phosphorylation of survivin on $\mathrm{Thr}^{34}$ and thus to its stability. Potential deregulation of these mitotic kinases may mediate the lack of survivin phosphorylation on $\mathrm{Thr}^{34}$ in PTX10 cells, leading to a compromised mitotic response to Taxol. At present, the exact mechanism by which survivin deregulation occurs is not clear. Nevertheless, our data show that survivin deregulation occurs during the development of Taxol resistance and that functional survivin is critical for the mitotic response to Taxol treatment. Additional studies are warranted to examine whether survivin deregulation may be associated with the clinical resistance to Taxol.

\section{REFERENCES}

1. Hayden JH, Bowser SS, Rieder CL. Kinetochores capture astral microtubules during chromosome attachment to the mitotic spindle: direct visualization in live newt lung cells. J Cell Biol 1990;111:1039-45.

2. Rusan NM, Fagerstrom CJ, Yvon AM, Wadsworth P. Cell cycle-dependent changes in microtubule dynamics in living cells expressing green fluorescent protein-alpha tubulin. Mol Biol Cell 2001;12:971-80.

3. Zhai Y, Kronebusch PJ, Simon PM, Borisy GG. Microtubule dynamics at the G2/M transition: abrupt breakdown of cytoplasmic microtubules at nuclear envelope breakdown and implications for spindle morphogenesis. J Cell Biol 1996;135:201-14.

4. Checchi PM, Nettles JH, Zhou J, Snyder JP, Joshi HC. Microtubule-interacting drugs for cancer treatment. Trends Pharmacol Sci 2003;24:361-5.

5. Jordan MA, Wilson L. Microtubules as a target for anticancer drugs. Nat Rev Cancer 2004;4:253-65.

6. Nogales E, Wolf SG, Downing KH. Structure of the alpha beta tubulin dimer by electron crystallography. Nature (Lond) 1998;391:199-203.

7. Snyder JP, Nettles JH, Cornett B, Downing KH, Nogales E. The binding conformation of Taxol in beta-tubulin: a model based on electron crystallographic density. Proc Natl Acad Sci USA 2001;98:5312-6. 
8. Schiff PB, Horwitz SB. Taxol stabilizes microtubules in mouse fibroblast cells. Proc Natl Acad Sci USA 1980;77:1561-5.

9. Derry WB, Wilson L, Jordan MA. Substoichiometric binding of taxol suppresses microtubule dynamics. Biochemistry 1995;34:2203-11.

10. Yvon AM, Wadsworth P, Jordan MA. Taxol suppresses dynamics of individual microtubules in living human tumor cells. Mol Biol Cell 1999;10:947-59.

11. Rowinsky EK. The development and clinical utility of the taxane class of antimicrotubule chemotherapy agents. Annu Rev Med 1997;48:353-74.

12. Gottesman MM, Fojo T, Bates SE. Multidrug resistance in cancer: role of ATPdependent transporters. Nat Rev Cancer 2002;2:48-58.

13. Giannakakou P, Sackett DL, Kang YK, et al. Paclitaxel-resistant human ovarian cancer cells have mutant beta-tubulins that exhibit impaired paclitaxel-driven polymerization. J Biol Chem 1997;272:17118-25.

14. Gonzalez-Garay ML, Chang L, Blade K, Menick DR, Cabral F. A beta-tubulin leucine cluster involved in microtubule assembly and paclitaxel resistance. J Biol Chem 1999;274:23875-82.

15. Kavallaris M, Kuo DY, Burkhart CA, et al. Taxol-resistant epithelial ovarian tumors are associated with altered expression of specific beta-tubulin isotypes. J Clin Investig 1997;100:1282-93.

16. Goncalves A, Braguer D, Kamath K, et al. Resistance to Taxol in lung cancer cells associated with increased microtubule dynamics. Proc Natl Acad Sci USA 2001;98: 11737-42.

17. Altieri DC. Validating survivin as a cancer therapeutic target. Nat Rev Cancer 2003;3:46-54.

18. Zhou J, Panda D, Landen JW, Wilson L, Joshi HC. Minor alteration of microtubule dynamics causes loss of tension across kinetochore pairs and activates the spindle checkpoint. J Biol Chem 2002;277:17200-8.
19. Zhou J, Gupta K, Yao J, et al. Paclitaxel-resistant human ovarian cancer cells undergo c-Jun NH2-terminal kinase-mediated apoptosis in response to noscapine. J Biol Chem 2002;277:39777-85.

20. Mabjeesh NJ, Escuin D, LaVallee TM, et al. 2ME2 inhibits tumor growth and angiogenesis by disrupting microtubules and dysregulating HIF. Cancer Cell 2003; 3:363-75.

21. Zhou J, Gupta K, Aggarwal S, et al. Brominated derivatives of noscapine are potent microtubule-interfering agents that perturb mitosis and inhibit cell proliferation. Mol Pharmacol 2003;63:799-807.

22. Giannakakou P, Poy G, Zhan Z, et al. Paclitaxel selects for mutant or pseudo-null p53 in drug resistance associated with tubulin mutations in human cancer. Oncogene 2000; $19: 3078-85$.

23. Cross SM, Sanchez CA, Morgan CA, et al. A p53-dependent mouse spindle checkpoint. Science (Wash D C) 1995;267:1353-6.

24. Zhou J, Yao J, Joshi HC. Attachment and tension in the spindle assembly checkpoint J Cell Sci 2002;115:3547-55.

25. O'Connor DS, Wall NR, Porter AC, Altieri DC. A p34(cdc2) survival checkpoint in cancer. Cancer Cell 2002;2:43-54.

26. Ling X, Bernacki RJ, Brattain MG, Li F. Induction of survivin expression by taxol (paclitaxel) is an early event, which is independent of taxol-mediated G2/M arrest. J Biol Chem 2004;279:15196-203.

27. Blagosklonny MV, Fojo T. Molecular effects of paclitaxel: myths and reality (a critical review). Int J Cancer 1999;83:151-6.

28. Li F, Ambrosini G, Chu EY, et al. Control of apoptosis and mitotic spindle checkpoint by survivin. Nature (Lond) 1998;396:580-4.

29. O'Connor DS, Grossman D, Plescia J, et al. Regulation of apoptosis at cell division by p34cdc2 phosphorylation of survivin. Proc Natl Acad Sci USA 2000;97:13103-7. 